\title{
Chemical potential and equations of state of hard core chain molecules
}

\author{
Fernando A. Escobedo and Juan J. de Pablo \\ Department of Chemical Engineering, University of Wisconsin-Madison, Madison, Wisconsin 53706-1691
}

(Received 6 February 1995; accepted 24 April 1995)

\begin{abstract}
A novel approach is presented for the development of equations of state for chain molecules. The basic assumptions of the approach are supported by results of computer simulations of the chemical potential of athermal chains. Our model establishes a bridge between some elements of Wertheim's thermodynamic perturbation theory and the generalized Flory theory. New equations of state are presented for freely jointed tangent hard-sphere chains which are shown to be more accurate than other existing equations. Extensions of our approach are also presented for branched polymers and blends of polymers. (C) 1995 American Institute of Physics.
\end{abstract}

\section{INTRODUCTION}

The development of equations of state for polymer systems is of fundamental importance to many industrial applications; accurate equations of state are needed for systematic correlation and prediction of the equilibrium thermodynamic properties of polymer liquids and alloys. The complexity of realistic polymeric systems has so far thwarted direct attempts to develop a completely statistical-mechanically based equations of state. A simplified model, which has provided important insights into the physics of polymers is the freely jointed hard chain model. An accurate equation of state for athermal chains would facilitate the development of equations of state for more realistic models, by providing the properties of a well-defined reference system for subsequent implementation of perturbation theory. Such a need has long been recognized and in recent years a number of equations of state have been proposed to describe the volumetric properties of hard chains. ${ }^{1-8}$

Hall and co-workers ${ }^{1,2}$ have developed the so-called generalized Flory (GF), Flory-Huggins, (GFH), and FloryDimer (GFD) theories based on the early models of Flory ${ }^{9}$ and Huggins ${ }^{10}$ for polymers on a lattice. The strategy of these theories consists of obtaining first a suitable, albeit approximate model for the probability of inserting a chain molecule into the system (insertion factor). This model is subsequently introduced into the osmotic equation of state, which rigorously relates the compressibility factor of the system to the insertion factor. In the GF and GFH theories, the insertion factor is estimated from knowledge of the insertion factor for a monomer into a monomer fluid; this result is then extrapolated to longer chains based on the concept of excluded volume. In the GFD theory, an improved estimate of the insertion probability is obtained by using approximate insertion factors derived from results for the monomer and dimer fluids.

The theory of associating fluids of Wertheim ${ }^{3,4}$ provides an alternative route for the development of equations of state for chain fluids. This theory relies on applying thermodynamic perturbation theory (TPT) to a polymerization process which proceeds by "bonding" monomer sites. In this manner, the Helmholtz free energy of the polymer system is expressed in terms of the Helmholtz free energy of the monomer fluid plus a term that represents the bonding contribution to the free energy of the system. In the first order theory (TPT1), the bonding term requires only knowledge of the pair correlation function of the monomer fluid. The TPT1 theory, initially intended to describe a polydisperse system with an average chain length $n$, was shown to also be applicable to a monodisperse $n$-mer system in the context of the statistical associating fluid theory (SAFT) of Chapman et al. ${ }^{5}$ An equation of state similar to that of the TPT1 theory has also been derived by Chiew. ${ }^{6}$ Chiew's approach is based on the solution of a particle-particle Ornstein-Zernicke integral equation subject to a Percus-Yevick closure and to connectivity constraints consistent with the chain geometry.

While all of these equations are relatively accurate when compared to computer simulation results, they all exhibit systematic deviations at both low and high densities. Relative errors tend to be larger at low densities and become more pronounced as the chain length increases. ${ }^{8,11,12}$ Several attempts have been made to improve the prediction of the theories mentioned above. A GF-trimer theory did not show any significant improvement over the GFD equation of state. ${ }^{10}$ Second-order TPT approximations (TPT2), which require the triplet correlation function of the monomer fluid, have also been developed. ${ }^{4,13}$ These equations are more accurate than the TPT1 and GFD equations of state, but their behavior at low and high densities is still unsatisfactory. Recently, the TPT1 theory has been extended (TPT1-D and SAFTD) by including information about the dimer fluid (i.e., the pair correlation function) ${ }^{7,8}$ This approach has resulted in equations that are more accurate than those derived by second-order perturbation theory. Further, they have the advantage of requiring more easily accessible information.

In this work we explore the similarities between the GF, GFD, TPT1, TPTD, and SAFTD theories at the level of the free energy. A careful analysis of computer simulation results for the chemical potential allows us to clearly define the common assumptions underlying these theories. A more general class of equations of state is formulated and its use is exemplified by deriving an equation analogous to TPTD or SAFTD but having improved accuracy. While all available theories perform an "extrapolation" of the volumetric properties of short (reference) chains to chains of arbitrary length, we propose an alternative "interpolation" scheme in which the volumetric properties of an infinitely long chain are built 
into the equation of state. The advantages of this approach are discussed.

In the next section we give a few thermodynamic manipulations to establish the relationships between the compressibility factor and the chemical potential. We then review the generalized versions of the GFD theory $\left(\mathrm{GF}-r^{\prime}\right)$ and TPT1-D theory (TPT1-r), which are in turn cast into chemical potential models. Subsequently, we present our more generalized equation of state and its applications for two particular reference fluid choices. Finally, we discuss some of the limitations and extensions of our approach to more complex systems such as mixtures of polymers or branched polymers.

\section{THERMODYNAMIC BACKGROUND}

Our starting point is the thermodynamic relationship between the pressure of a one-component system consisting of $N, n$-mer molecules and its Helmholtz free energy,

$$
P=-\left.\frac{\partial A}{\partial V}\right|_{T, N} .
$$

It is convenient to introduce the packing fraction, $\eta$, as the integration variable

$$
\eta=v_{n} \rho=\frac{\pi}{6} \sigma^{3} n \frac{N}{V}
$$

where $\sigma$ is the diameter of a monomer unit or site, $v_{n}$ is the molecular volume of each $n$-mer, and $\rho$ the site-number density. Equation (1) can then be rewritten as

$$
Z=\left.\eta \frac{\partial\left(\frac{\beta}{N} A\right)}{\partial \eta}\right|_{\beta, N},
$$

where $\beta=\left(k_{B} T\right)^{-1}\left(k_{B}\right.$ is Boltzmann's constant). Equation (3) is integrated to give

$$
\frac{\beta}{N} A(\eta)-\frac{\beta}{N} A(0)=\int_{\eta^{\prime}=0}^{\eta} \frac{Z}{\eta^{\prime}} d \eta^{\prime}
$$

An analogous equation can be written for the ideal gas (with $Z=1$ ). Such an equation is subtracted from Eq. (4) to obtain a relationship for the excess (or residual) Helmholtz free en$\operatorname{ergy} A^{E}$ (real fluid property minus the ideal fluid value)

$$
\frac{\beta}{N} A^{E}(\eta)-\frac{\beta}{N} A^{E}(0)=\int_{\eta^{\prime}=0}^{\eta} \frac{Z-1}{\eta^{\prime}} d \eta^{\prime} .
$$

The value of $A^{E}(0)$ will depend on the choice of the reference state. In our subsequent discussion, the ideal gas free energy includes contributions from intramolecular interactions, $A^{\text {intra }}$,

$$
\frac{\beta}{N} A^{\mathrm{id}}(\eta)=\ln \left(\frac{\eta}{v_{n}} \Lambda^{3}\right)-1+\frac{\beta}{N} A^{\text {intra }}
$$

In that case we can set $A^{E}(0)=0$ in Eq. (5). The chemical potential is obtained from

$$
\beta \mu^{E}=\left.\frac{\partial\left[N\left(\frac{\beta}{N} A^{E}\right)\right]}{\partial N}\right|_{\beta, V}=\frac{d}{d \eta}\left[\eta \frac{\beta}{N} A^{E}(\eta)\right],
$$

$$
\beta \mu^{E}=\int_{\eta^{\prime}=0}^{\eta} \frac{Z-1}{\eta^{\prime}} d \eta^{\prime}+Z-1 .
$$

Thus, given an equation of state, Eq. (8) allows us to compute the excess chemical potential at any given density. It is also desirable to express explicitly the compressibility factor as a function of the excess chemical potential. Equation (8) (premultiplied by $\eta$ ) can be differentiated and further simplified to give

$$
d(\eta Z-\eta)=\eta \beta d \mu^{E} .
$$

Integration by parts within the limits $[0, \eta]$ gives

$$
Z=1+\beta \mu^{E}-\frac{\beta}{\eta} \int_{\eta^{\prime}=0}^{\eta} \mu^{E} d \eta^{\prime} .
$$

This equation can be immediately identified with the socalled "osmotic equation of state" ${ }^{1}$ by recalling the relationship between the chemical potential and the insertion factor, $p(n)$, which measures the probability of inserting a whole chain of length $n$ into a fluid of $n$-mers at a given packing fraction $\eta$ :

$$
\ln p(n)=-\beta \mu^{E}(n) .
$$

The osmotic equation of state has been previously derived following different arguments by Dickman and Hall. ${ }^{1}$ Equation (10) is particularly convenient when applied to mixtures, where the alternative approach becomes much more involved. ${ }^{14,15}$ For mixtures we replace $\mu^{E}$ in Eq. (10) by the excess molar Gibbs free energy $g^{E}$ of the mixture,

$$
\mu^{E}(\text { mixture })=g^{E}=\sum_{i=1}^{N_{c}} x_{i} \mu^{E}\left(n_{i} \mid m\right),
$$

where $N_{c}$ is the number of components in the mixture, $x_{i}$ is the molar fraction of species $i$ in the system, and $\mu^{E}\left(n_{i} \mid m\right)$ is the chemical potential associated with the insertion of a molecule of species $i$ (of chain-length $n_{i}$ ) into the mixture $m$. Substituting $\mu^{E}$ (mixture) into Eq. (10) we arrive at

$$
\begin{aligned}
Z(\text { mixture })= & 1+\sum_{i=1}^{N_{c}} x_{i} \beta \mu^{E}\left(n_{i} \mid m\right) \\
& -\frac{1}{\eta} \sum_{i=1}^{N_{c}} x_{i} \int_{\eta^{\prime}=0}^{\eta} \beta \mu^{E}\left(n_{i} \mid m\right) d \eta^{\prime} .
\end{aligned}
$$

By resorting to a suitable redefinition for the insertion factor, this equation translates to the alternate osmotic equation of state derived by Wichert and Hall ${ }^{15}$ for a binary mixture (obtained through a simultaneous insertion of both species into the fluid).

\section{THE GENERALIZED FLORY-( $\boldsymbol{r}^{\prime}$-MER) THEORY}

The basic assumption invoked by the generalized theory of Honnell and Hall ${ }^{2}$ is that the probability of inserting a $j$-mer into the $n$-mer fluid is approximately equal to $p(j)$, which is the probability of inserting a $j$-mer into a $j$-mer fluid. This approximation is applied to two reference fluids $j=r^{\prime}-1$ and $j=r^{\prime}\left(r^{\prime}<n\right)$. The relationship between the probability of adding a monomer to a growing chain of 
TABLE I. Constants for the equation of state model, Eq. (17) for different freely jointed tangent hard-sphere chain systems.

\begin{tabular}{ccccccc}
\hline \hline $\begin{array}{l}n \text {-mer } \\
\text { system }\end{array}$ & \multicolumn{1}{c}{$c_{1}$} & $c_{2}$ & $c_{3}$ & $\begin{array}{c}\text { Source } \\
\text { data } \\
\text { (Ref.) }\end{array}$ & $\begin{array}{c}\text { Source } \\
\text { constants } \\
\text { (Ref.) }\end{array}$ & $\begin{array}{c}\text { Avg. \% } \\
\text { Error }\end{array}$ \\
\hline 1 & 1.0 & 1.0 & -1.0 & 16 & 16 & \\
2 & 2.45696 & 4.10386 & -3.75503 & 17 & 17 & 0.29 \\
3 & 3.14921 & 8.94817 & -6.18837 & 12,23 & This work & 1.0 \\
4 & 4.25001 & 13.98270 & -12.19938 & 8 & This work & 0.32 \\
8 & 7.88859 & 32.25721 & -25.90072 & 8 & This work & 0.29 \\
16 & 13.50565 & 79.10375 & -64.56661 & 8,11 & This work & 0.64 \\
\hline \hline
\end{tabular}

length $k\left(r^{\prime} \leqslant k \leqslant n\right)$ and the probability of adding a monomer to the $\left(r^{\prime}-1\right)$-mer to form the $r^{\prime}$-mer is estimated from

$$
P(k+1 \mid k) \approx\left[P\left(r^{\prime} \mid r^{\prime}-1\right)\right]^{f} \approx\left[\frac{p\left(r^{\prime}\right)}{p\left(r^{\prime}-1\right)}\right]^{f} .
$$

The exponent $f$ accounts for the fact that the effective free volume required to insert the $(k+1)$ th monomer can be different from that for the $r^{\prime}$ th monomer,

$$
f=\frac{v_{e}(k+1)-v_{e}(k)}{v_{e}\left(r^{\prime}\right)-v_{e}\left(r^{\prime}-1\right)},
$$

where $v_{e}\left(r^{\prime}\right)$ is the excluded volume of an $r^{\prime}$-mer molecule. The insertion factor for a whole $n$-mer molecule (into the $n$-mer fluid) is then estimated as the product of the probability of first inserting the $\left(r^{\prime}-1\right)$-mer, and the conditional probabilities [Eq. (13)] of growing the chain from the $\left(r^{\prime}-1\right)$-mer to the $n$-mer (site by site). The resulting equation can be expressed as

$$
p(n)=p\left(r^{\prime}-1\right)^{-Y_{n, r^{\prime}}} p(r)^{Y_{n, r^{\prime}+1}},
$$

where

$$
Y_{n, r^{\prime}}=\frac{v_{e}(n)-v_{e}\left(r^{\prime}\right)}{v_{e}\left(r^{\prime}\right)-v_{e}\left(r^{\prime}-1\right)} .
$$

When Equation (14) is introduced into the osmotic equation, the following equation can be obtained relating the compressibility factors of the reference fluids $\left[r^{\prime}\right.$-mer and $\left(r^{\prime}-1\right)$-mer $]^{2}$

$$
Z(n)=\left(1+Y_{n, r^{\prime}}\right) Z\left(r^{\prime}\right)-Y_{n, r^{\prime}} Z\left(r^{\prime}-1\right) .
$$

Equation (16) for $r^{\prime}=2$ yields the GF-Dimer (GFD) equation of state, for which the equations of state of the monomer and the dimer fluids are necessary. Honnell and Hall used the equations of Carnahan-Starling ${ }^{16}$ and Tildesley-Streett ${ }^{17}$ to calculate $Z(1)$ and $Z(2)$, respectively. These two equations are of the form

$$
Z(i)=\frac{1+c_{1} \eta+c_{2} \eta^{2}+c_{3} \eta^{3}}{(1-\eta)^{3}} .
$$

The constants required by Eq. (17) are given in Table I for various values of $i$.

\section{WERTHEIM'S TPT THEORY AND ITS VARIATIONS}

Wertheim studied a graphical density expansion of the Helmholtz free energy for associating fluids modeled as hard spherical molecules with one or two "sticky" sites on their surface. In the limit of association, the spheres form chain molecules. Using thermodynamic perturbation theory, Wertheim showed that to first order (considering singleassociating-bond graphs only) the free energy of an $n$-mer fluid $^{4}$ (or a fluid with number average chain length $=n$ ) ${ }^{5}$ is given by

$$
\frac{\beta A^{E}(n)}{N}=\frac{\beta A^{E}(1)}{N}-(n-1) \ln g_{1}(\sigma),
$$

where $A^{E}(n)$ and $A^{E}(1)$ are the excess Helmholtz free energies of the chain and the monomer fluids, respectively, and $g_{1}(\sigma)$ is the pair correlation function of the monomer fluid evaluated at the bond length $\sigma$ and at the same packing fraction. The first term on the right-hand side of Eq. (18) represents the contribution to the free energy due to the unbonded fluid; the second term corresponds to the contribution (perturbation) due to formation of bonds. The compressibility factor derived from Eq. (18) is therefore

$$
Z_{\mathrm{TPT} 1}(n)=n Z(1)-(n-1)\left[1+\eta \frac{\partial \ln g_{1}(\sigma)}{\partial \eta}\right] .
$$

The compressibility factor of the monomer fluid is related to $g_{1}(\sigma)$ through the pressure equation

$$
g_{1}(\sigma)=\frac{1}{4 \eta}[Z(1)-1] .
$$

In the second-order version of Wertheim's theory (TPT2), a correction term is added to Eq. (18) by considering higher-order graphs containing two associating bonds, thereby yielding a more accurate equation of state. ${ }^{4,13}$ The correction required depends on the triplet correlation function of the hard sphere fluid. Given that additional empirical information has to be built into the model (i.e., the triplet correlation function) to improve TPT1, a number of alternative routes have been devised to arrive at more accurate equations of state while resorting to more easily accessible empirical information. ${ }^{7,8,18}$ In the spirit of Honnell and Hall's theory, the TPT1 theory could be refined by incorporating information from the dimer fluid. Two such attempts have been recently presented by Ghonasgi and Chapman ${ }^{7}$ and Chang and Sandler. ${ }^{8}$ The equations of state derived by these authors are essentially identical, although the arguments and empirical models adopted by these authors are somewhat different. For brevity, we review the approach of Chang and Sandler only. These authors modified the equation for the free energy as follows:

$$
A^{E}(n)=A^{E}(1)+\frac{n N}{2} A_{1}^{\text {bond }}+\frac{(n-2) N}{2} A_{2}^{\text {bond }},
$$

where $A_{1}^{\text {bond }}$ is the free energy change due to the formation of monomer-monomer bonds ( $n N / 2$ total bonds) and $A_{2}^{\text {bond }}$ is the change in free energy produced by bonding the dimers until chains of length $n$ are formed [(n-2)N/2 total bonds]. Using the definition of a potential of mean force $(\omega)$ they write

$$
\beta \omega_{j}=\beta A_{j}^{\text {bond }}=-\ln g_{j}(\sigma),
$$


where $g_{j}(\sigma)$ is the site-site correlation function at contact for the $j$-mer fluid. The resulting compressibility factor can be compactly expressed using a more general equation of state based on an initial mixture of $r$-mers ${ }^{7}$

$$
Z(n)=\frac{n}{r} Z^{*}(r)-\left(\frac{n}{r}-1\right)\left[1+\eta \frac{\partial \ln g_{r}(\sigma)}{\partial \eta}\right]
$$

and setting $r=2$ [the expression required for $Z^{*}(2)$ is found by first applying Eq. (23) for $n=2, r=1]$.

Originally, Ghonasgi and Chapman ${ }^{7}$ and Chang and Sandler ${ }^{8}$ used an approximate expression for $g_{2}(\sigma)$ derived by Chiew ${ }^{19}$ from integral-equation theory. Both groups of researchers found, however, that more accurate results could be attained by relying on fits of $g_{2}(\sigma)$ to simulation data. Chang and Sandler adopted the result of Yethiraj and Hall ${ }^{20}$

$$
g_{2}^{\mathrm{TPT} 1-\mathrm{D}}(\sigma)=\frac{(2-\eta)(0.534+0.414 \eta)}{2(1-\eta)^{3}}
$$

while Ghonasgi and Chapman corrected Chiews's equation to get

$$
g_{2}^{\text {SAFT-D }}(\sigma)=\frac{1+2 \eta+26.45031 \eta^{6.17}}{2(1-\eta)^{2}} .
$$

Chang and Sandler ${ }^{8}$ compared their equation, TPT1-D, and TPT2 to computer simulation results for 4-mer, 8-mers, and 16-mers and found that TPT1-D is slightly superior to TPT2. As shown latter, a comparison of TPT1-D with the equation of Ghonasgi and Chapman (SAFTD) shows that both equations give comparable results, the latter being slightly more accurate.

\section{GENERAL MODELS FOR THE CHEMICAL POTENTIAL AND EQUATIONS OF STATE}

In the context of the GF- $r$ theory, the equation for the insertion factor (12) can be translated into a relation of chemical potentials

$$
\mu^{E}(n)=-Y_{n, r^{\prime}} \mu^{E}\left(r^{\prime}-1\right)+\left(Y_{n, r^{\prime}}+1\right) \mu^{E}\left(r^{\prime}\right) .
$$

Accurate estimates of $v_{e}(n)$ (required to evaluate $Y_{n, r^{\prime}}$ ) could be found from $\left(r^{\prime \prime}>2\right)^{2}$

$$
v_{e}(n)=v_{e}\left(r^{\prime \prime}\right)+\left(n-r^{\prime \prime}\right)\left[v_{e}\left(r^{\prime \prime}\right)-v_{e}\left(r^{\prime \prime}-1\right)\right] .
$$

To date, this relation has been extensively used with $r^{\prime \prime}=3$. If we set $r^{\prime}=r^{\prime \prime}\left(r^{\prime}>2\right)$, Eqs. (15) and (27) yield $Y_{n, r^{\prime}}$ $=n-r^{\prime}$, and if we introduce $r=r^{\prime}-1$, Eq. (26) can be written as follows:

$$
\mu^{E}(n)=\mu^{E}(r)+(n-r)\left[\mu^{E}(r+1)-\mu^{E}(r)\right] ; \quad r>1 .
$$

It is important to note that this equation is consistent with the GF- $(r+1)$ theory but, since $r>1$, it cannot be reduced to the GFD theory.

In the context of the TPT1 $-r$ theory, Eq. (23) can be cast in terms of chemical potentials [by means of Eq. (8)] as follows:

$$
\mu^{E}(n)=\mu^{E}(r)+(n-r) \frac{\mu^{E}(r)+\mu_{\text {bond }}^{E}(r)}{r},
$$

where we have defined

$$
\beta \mu_{\text {bond }}^{E}(r)=-\frac{\partial}{\partial \eta}\left[\eta \ln g_{r}(\sigma)\right] .
$$

Equations (28) and (29) allow us to clearly compare the approximations underlying the GF- $r$ and TPT1- $r$ theories. Both theories basically invoke the following approximation:

$\mu^{E}($ grow $n$-mer $)=\mu^{E}($ grow $r$-mer $)$

$$
+\mu^{E} \text { [append }(n-r) \text { sites to } r \text {-mer] }
$$

$$
\begin{aligned}
\approx & \mu^{E}(\text { grow } r \text {-mer }) \\
& +(n-r) \mu^{E}(\text { append a monomer }) .
\end{aligned}
$$

In both cases the "growing" and "appending" processes should take place in the $n$-mer fluid. An additional approximation introduced by the theories is the use of a convenient reference host system for these processes in lieu of the $n$-mer fluid. The basic difference between the two theories resides in the manner in which they estimate the chemical potential associated with appending every monomer until an $n$-mer is completed:

GF- $r^{\prime}: \quad \mu^{E}$ (append a monomer)

$$
\approx\left[\mu^{E}(r+1)-\mu^{E}(r)\right],
$$

TPT1-r: $\quad \mu^{E}$ (append a monomer)

$$
\approx\left[\frac{\mu^{E}(r)+\mu_{\text {bond }}^{E}(r)}{r}\right] \text {. }
$$

In both cases " $\mu^{E}$ (append a monomer)" is assumed to be independent of the length of the growing chain. If we now introduce the approximation that $\mu_{\text {bond }}^{E}(r)=\left[\mu^{E}(2 r)\right.$ $\left.-2 \mu^{E}(r)\right]$, a more general expression for $\mu^{E}(n)$ can be written

$$
\mu^{E}(n)=\mu^{E}(r)+\frac{n-r}{r^{*}-r}\left[\mu^{E}\left(r^{*}\right)-\mu^{E}(r)\right] .
$$

The GF- $r^{\prime}$ and TPT1 $r$ are particular cases of Eq. (35) obtained by setting $r^{*}=r+1$ or $r^{*}=2 r$, respectively [provided that, for the TPT1 $-r, \mu^{E}\left(r^{*}\right)$ is computed from Eqs. (29) to (30) with $n \rightarrow r^{*}$.

Recently, we have reported accurate simulation results for the incremental chemical potential of hard chains at various densities. ${ }^{21}$ Some of these results are given in Fig. 1. It is shown that Eq. (32) is fairly accurate for $r \geqslant 2$, i.e., the incremental chemical potential becomes essentially constant after the first three or four segments have been introduced. It is also shown that the values of $\Delta \mu^{E}=\mu^{E}$ (append a monomer) are, to a good approximation, independent of the chain length of the system. The accuracy of these approximations varies slightly depending on the chain length and density of the system.

Some general observations are in order.

(1) Although greater accuracy can be achieved adopting a longer reference system to evaluate the " $\mu^{E}$ (grow $r$-mer)" term in Eq. (32) (provided that its properties are 


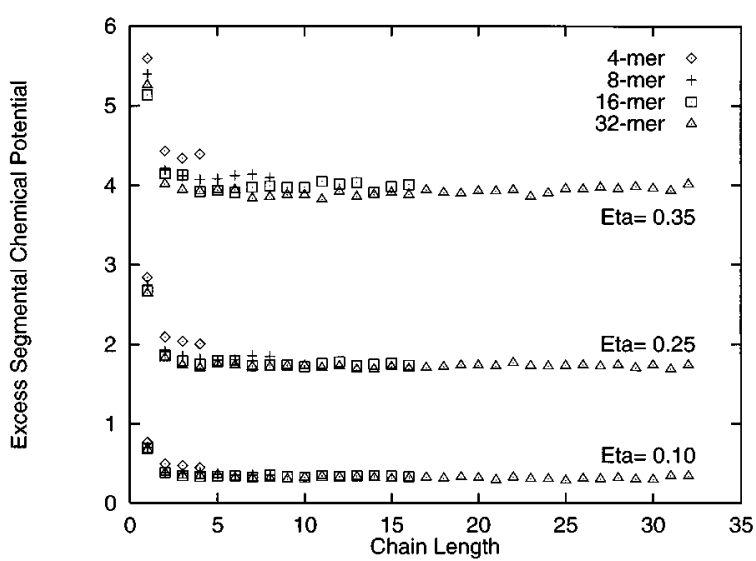

FIG. 1. Segmental excess chemical potential in reduced units, $\beta \Delta \mu^{E}$, for freely jointed hard-sphere chains of different lengths at low and intermediate densities $(\eta=0.1,0.25,0.35)$. The symbols show results of Monte Carlo simulations (Ref. 21).

taken from an accurate equation of state), it is the " $\mu^{E}$ (append a monomer)" term which will largely determine the overall accuracy of the theory for large $n$. In estimating the latter term, a value of $r \geqslant 2$ seems advisable.

(2) As noted in various studies, ${ }^{1,2,14}$ an important drawback of the assumption " $\mu^{E}$ (grow an $r$-mer in the $n$-mer fluid) $\approx \mu^{E}$ (grow an $r$-mer in the $r$-mer fluid)" is that the chemical potential is overestimated, particularly at low densities (in Fig. 1, the relative differences between the curves is larger at $\eta=0.10$ ). The reason is that the local site density of the $n$-mer fluid is more heterogeneous than that of the $r$-mer fluid, which makes it easier to "grow" the $r$-mer in the $n$-mer fluid.

To evaluate the " $\mu^{E}$ (append a monomer)" term, we must invariably resort to some sort of numerical data (computer simulation results) and, pragmatically, the choice of the specific information employed is just a matter of convenience. The "amount" of empirical information required can, of course, be minimized by introducing further approximations into the model. However, the merits of such an approach are dubious because savings in empirical input often translate into a further loss of accuracy, even if the approximations introduced have solid, theoretical grounds. Three obvious sources of empirical data are.

(1) Correlations of compressibility factors for homologous but "shorter" chain molecules. A variety of efficient simulation methods are available to calculate PVT properties of model fluids. For the case of freely jointed tangent hard-sphere chains a number of studies $8,11,12,22,23$ have provided PV data for various chain lengths. In Table I we summarize a few correlations based on the reported data. The data has been fitted to model (17).

(2) Correlations of structural properties such as the site correlation function of reference fluids [i.e., Eq. (19)].

(3) Free energy or chemical potential data. This is, of course, the most straightforward method to evaluate the required term.
The main reason why the chemical potential "route" has not been extensibly exploited in the past has probably been due to the difficulties encountered in computing chemical potentials using conventional simulation methods (particularly for dense, chainlike fluids). However, a new method that overcomes these difficulties has been recently developed. ${ }^{21}$ Such a method will be even more relevant for the study of other intermolecular potential models for which PVT data are scarce.

\section{ALTERNATIVE EQUATIONS OF STATE}

It is beyond the scope of this note to explore the numerous possibilities to estimate a correlation for $\mu^{E}$ (append a monomer) in Eq. (32). We shall illustrate how the accuracy of the resulting equation of state can be improved based on a judicious estimate of the incremental chemical potential based on correlations of $Z(r)$ for suitable reference fluids. The approximation for $\mu^{E}$ of Eq. (35) can be recast in terms of compressibility factors by invoking Eq. (10); the result is

$$
Z(n)=Z(r)+\frac{n-r}{r^{*}-r}\left[Z\left(r^{*}\right)-Z(r)\right] .
$$

Equation (36) includes the GF- $r$ and TPT1- $r$ theories as particular cases, provided that a suitable translation of terms is carried out. For this purpose, we point out again that in the context of the TPT1-r theory (where $r^{*}=2 r$ ), the $Z(r)$ and $Z(2 r)$ terms required are not generally evaluated from a direct correlation of the PVT properties of the corresponding fluids. Instead, they are obtained via a recursive approach [Eqs. (29) and (30)] in which

$$
Z(2 k)=2 Z(k)-1-\eta \frac{\partial}{\partial \eta}\left[\ln g_{k}(\sigma)\right], \quad k=1,2, \ldots, r .
$$

Correlations for $Z(1)$ and $g_{1}(\sigma), g_{2}(\sigma), \ldots, g_{r}(\sigma)$ are therefore needed as an input for the model.

On the other hand, it has already been noted that the GFD does not fall into this scheme because Eq. (27), used to cast the GF- $r^{\prime}$ into our general model, is not accurate for $r^{\prime \prime}=2$ (that is, $r=1$ ). This sole fact should suffice to explain why the GFD theory is more satisfactory than the plain TPT1 theory, which is obtained by setting $r=1$ and $r^{*}=2$ in Eq. (36).

We expect Eq. (36) to become more accurate for larger $r^{*}$ (closer to $\left.n\right)$. Therefore $r^{*}=r+1$ seems cruder than $r^{*}=2 r$ (for fixed $r$ ). It has been noted before that an implementation of the GF-Trimer equation of state did not offer any conclusive advantages over the original GFD equation. ${ }^{12}$ We have also explored the GF-Tetramer and found that the improvement achieved over the GFD equation is marginal (see Table III).

\section{A. A dimer-tetramer equation}

Equation (36) can be rewritten as

$$
Z(n)=\frac{n}{r} Z(r)+\left(\frac{n}{r}-1\right) Z_{r-r}^{\text {bond }}
$$

where $Z_{r-r}^{\text {bond }}=\left[r Z\left(r^{*}\right)-r^{*} Z(r)\right] /\left(r^{*}-r\right)$. 
A simple phenomenological interpretation can be provided by means of the following "polymerization" path

(1) Begin with a system of $(n N / r) r$-mers at constant $V$ (and $T$ ) at the equilibrium pressure $P(r, \eta)$.

(2) (2N) $r$-mers polymerize to form $N, 2 r$-mers in solution with the remaining $(n N / r-2 N) r$-mers. At constant volume (constant $\eta$ ) the system experiences a pressure reduction of magnitude $N \Delta P_{r-r}^{\text {bond }}$.

(3) A new polymerization step is carried out, this time to form a blend of $N, 3 r$-mers and the remaining $r$-mers. The observed pressure depression is now $N \Delta P_{2 r-r}^{\text {bond }}$.

(4) The process is repeated until all $r$-mers are depleted to form $N, n$-mers and the system reaches the pressure $P(n, \eta)$.

Equation (38) can be obtained for this process if we assume that the pressure depression (per bond) at each stage $k, \Delta P_{k r-r}^{\text {bond }}$, is always the same (i.e., independent of $k$ and the composition of the system at that point). The model, however, is essentially exact for $n=2 r$ because only one type of bond is formed; the bonding contribution to the pressure of the system is $\Delta P_{r-r}^{\text {bond }}$ for every bond. This fact can be capitalized to estimate $Z_{r-r}^{\text {bond }}$ from Eq. (38) by setting $n=2 r$; since it has been postulated that $r$ should be at least equal to 2 , the final equation of state takes the form

$$
\begin{aligned}
Z(n) & =\frac{n}{2} Z(2)+\left(\frac{n}{2}-1\right)[Z(4)-2 Z(2)] \\
& =Z(2)+\frac{(n-2)}{2}[Z(4)-Z(2)] .
\end{aligned}
$$

Equation (39) is consistent with Eq. (36) and constitutes, in essence, an alternative way of writing the TPT1-Dimer theory. Equation (39) has been implemented using the constants of Table I for the correlations of $Z(2)$ and $Z(4)$ as given by Eq. (17).

\section{B. An interpolating equation of state}

With the convention that $n>r$, Eq. (36) basically provides an extrapolating formula whenever $n>r^{*}$. However, it could also be used as an interpolation formula if $r^{*}>n$. A convenient choice of reference fluid for this purpose would be a fluid of infinitely long chains, i.e., $r^{*} \rightarrow r_{\infty}$. If we postulate that it is the lack of chain ends that will determine the "packing" properties of the $r_{\infty}$-mer fluid, then its volumetric properties could be approximately obtained by simulating a sufficiently long polymer (or a ring). We have performed NPT simulations for systems of 600-mer and 3000-mer chains and found that the compressibility factors based on the monomer density, $Z(n) / n$, are essentially identical for both systems (within the statistical uncertainty). These results suggest that a limiting behavior has been attained for the compressibility of the system. Given the small number of chains employed for these calculations, our results (particularly those for configurational properties) could suffer from finite size effects. Note, however, that simulations on systems of smaller freely joined chains indicate that the total pressure of the system is relatively insensitive to the number of molecules employed in the calculation (as long as the total
TABLE II. Simulation results for the $r_{\infty}$-mer hard-sphere chains. The MC steps correspond to the averaging part of the calculation and apply to each of two independent simulations.

\begin{tabular}{lccc}
\hline \hline$\frac{6}{\pi} P^{*}$ & $\eta$ & Vol. moves & MC steps \\
\hline 0.0063 & $0.053 \pm 0.002$ & $250 \times 10^{3}$ & $500 \times 10^{6}$ \\
0.036 & $0.103 \pm 0.002$ & $250 \times 10^{3}$ & $500 \times 10^{6}$ \\
0.11 & $0.153 \pm 0.003$ & $200 \times 10^{3}$ & $500 \times 10^{6}$ \\
0.26 & $0.203 \pm 0.003$ & $200 \times 10^{3}$ & $500 \times 10^{6}$ \\
0.54 & $0.253 \pm 0.003$ & $200 \times 10^{3}$ & $500 \times 10^{6}$ \\
1.01 & $0.302 \pm 0.002$ & $200 \times 10^{3}$ & $600 \times 10^{6}$ \\
1.80 & $0.351 \pm 0.002$ & $200 \times 10^{3}$ & $600 \times 10^{6}$ \\
3.10 & $0.401 \pm 0.002$ & $300 \times 10^{3}$ & $800 \times 10^{6}$ \\
5.20 & $0.450 \pm 0.002$ & $300 \times 10^{3}$ & $800 \times 10^{6}$ \\
\hline \hline
\end{tabular}

number of sites in the system is large enough). In Table II we present the results of NPT simulations performed on a single-chain system of 3000 beads. Since conventional NPT simulations become highly inefficient to generate volume fluctuations at high densities $(\eta>0.25)$, for dense packings we employed the slab-volume moves recently developed by us. ${ }^{24}$ These results have been fitted to the following model:

$$
\frac{P^{*}\left(r_{\infty}\right)}{\eta}=\frac{\left(0.81255 \eta+5.05646 \eta^{2}-4.11943 \eta^{3}\right)}{(1-\eta)^{3}}
$$

where the reduced pressure of the system is given by $P^{*}=\pi / 6 \sigma^{3} \beta P$. For an $r^{*}$-mer fluid we have $P^{*} / \eta=Z\left(r^{*}\right) / r^{*}$ and, in the limit of $r^{*} \rightarrow r_{\infty}$, Eq. (36) takes the form

$$
Z(n)=Z(r)+(n-r)\left[\frac{P^{*}\left(r_{\infty}\right)}{\eta}\right] .
$$

This equation is expected to become increasingly accurate as $n$ becomes larger. Since very long chains are of great practical interest, Eq. (41) should be particularly useful for practical applications.

Figure 2 shows $Z / n$ as a function of $\eta$ from simulation data of Chang and Sandler ${ }^{8}$ for $n=4,8,16$, and our own simulation results for $n=16,32,51$, and the $r_{\infty}$-mer fluid. ${ }^{11}$ The equations of state for the monomer ${ }^{16}$ and the dimer ${ }^{17}$ are also included. It is observed that simulation data for any finite-size chain lies within the curves corresponding to the monomer and the $r_{\infty}$-mer fluid. The fact that all curves follow an analogous trend further supports the use of an extraor interpolation scheme such as that given by Eq. (36).

\section{Comparative results}

We have compared the predictions of Eqs. (39) and (41) with the computer simulations results of Chang and Sandler ${ }^{8}$ for $n=4,8,16$ and our own simulation results for $n=16$, 32, and 51. ${ }^{11}$ This comparison is summarized in Table III along with the corresponding results obtained using other equations of state. In most cases Eq. (39) gives the smallest average relative error, closely followed by Eq. (41) and the SAFTD equation. The results presented for Eq. (41) correspond to $r=2$ and as expected, the accuracy improves as $n$ is increased; for 51-mers it gives better results than all other 


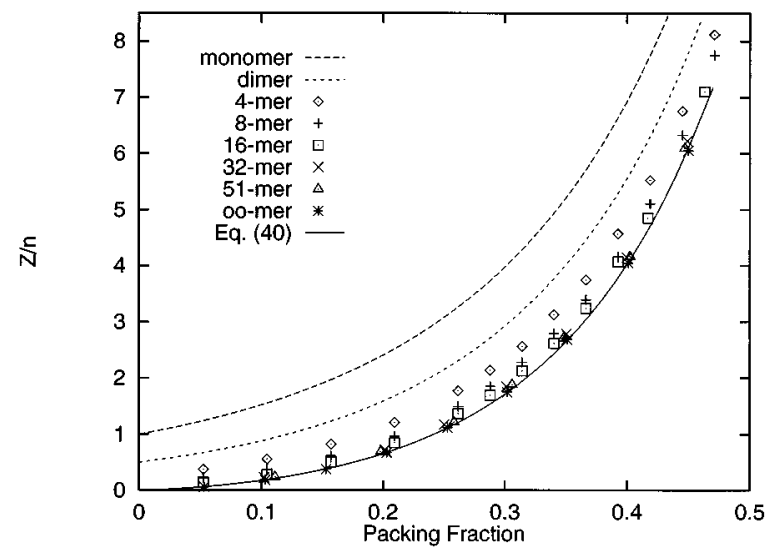

FIG. 2. Compressibility factor per chain segment vs packing fraction for freely jointed tangent hard-sphere systems of various chain lengths. The symbols show results of simulations from Refs. 8, 11, and this work. The equations of state for the monomer (Ref. 16), the dimer (Ref. 17), and the infinitely long chain [Eq. (40)] are shown by the lines.

equations. The choice of $r$ also affects the accuracy of Eq. (41); the overall average error (for all systems) is $1.8 \%$, $1.1 \%$, and $1.0 \%$ for $r=1,2$, and 4 , respectively.

Our simulation results for the 32-mer system are plotted in Fig. 3 along with the predictions of Eq. (39). Excellent agreement between simulation data and Eq. (39) is observed for the entire range of densities. Table IV shows a detailed comparison of various equations of state for 32-mers. It is observed that, for all equations of state with the exception of Eq. (41), the larger relative deviations from the simulation occur in the low density range $(\eta<0.25)$. In fact, this qualitative trend of overpredicting the compressibility factor at low density is also observed for all the chain-length systems considered in Table III, the deviations being larger for longer chains. As explained earlier, this feature is the result of overestimating the chemical potential associated with approximating the $n$-mer host system by a shorter chain length system for the inserting and appending processes.

To explore in more detail the performance of the equations of state at low densities, we compare their corresponding second virial coefficients to the simulation data of Yethiraj et al. ${ }^{26}$ For equations of state which can be reduced to the form given by Eq. (17) (having constants $c_{1}, c_{2}$, and $c_{3}$ dependent on $n$ ), the second virial coefficient is related to $c_{1}$ as follows:

TABLE III. Average relative error (\%) in the compressibility factor of freely jointed tangent hard-sphere chain systems from various theories.

\begin{tabular}{rccccccc}
\hline \hline$n$-mer & GFD & GF-(4-mer) & TPT1 & TPT1-D & SAFTD & Eq. (39) & Eq. (41) \\
\hline 3 & 2.76 & 1.05 & 2.83 & 2.10 & 2.12 & 1.82 & 1.39 \\
4 & 1.70 & 0.34 & 2.57 & 0.77 & 0.73 & 0.34 & 0.72 \\
8 & 3.57 & 2.37 & 5.33 & 1.78 & 1.26 & 0.48 & 0.88 \\
16 & 6.00 & 4.75 & 7.83 & 3.29 & 2.26 & 1.26 & 1.20 \\
32 & 6.50 & 5.67 & 9.29 & 3.40 & 2.49 & 1.08 & 1.12 \\
51 & 6.90 & 6.15 & 9.93 & 3.87 & 2.78 & 1.53 & 1.24 \\
\hline \hline
\end{tabular}

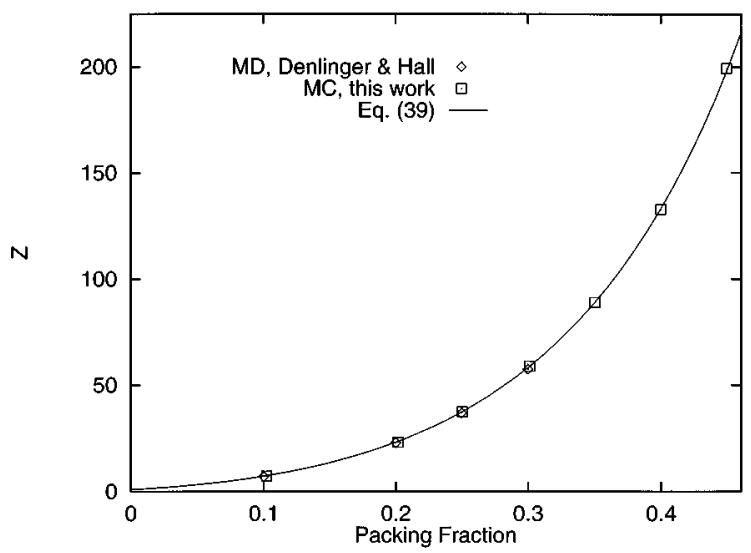

FIG. 3. Compressibility factor vs packing fraction for freely jointed tangent hard-sphere chains of 32 segments. The diamonds show results of simulations by Denlinger and Hall (Ref. 25), and the squares show results of simulations performed in this work. The line shows the predictions of Eq. (39).

$$
B_{2}=\frac{\pi}{6} \sigma^{3} n\left(3+c_{1}\right) .
$$

For Eq. (36), with $Z(r)$ and $Z\left(r^{*}\right)$ expressed as in Eq. (17), we can write

$$
\begin{aligned}
\frac{6}{\pi \sigma^{3} n^{2}} B_{2}= & \left(\alpha_{0}+\frac{\alpha_{1}}{n}\right) \\
= & {\left[\frac{c_{1}\left(r^{*}\right)-c_{1}(r)}{r^{*}-r}\right.} \\
& \left.+\frac{3\left(r^{*}-r\right)+r^{*} c_{1}(r)-r c_{1}\left(r^{*}\right)}{\left(r^{*}-r\right) n}\right] .
\end{aligned}
$$

For Eq. (39) $\alpha_{0}=0.8965, \alpha_{1}=3.664$ and for Eq. (41) $(r$ =2) $\alpha_{0}=0.8126, \alpha_{1}=3.832$. For the other equations constants $\alpha_{0}$ and $\alpha_{1}$ can be found elsewhere. ${ }^{7,8}$ Figure 4 shows that, overall, SAFTD and Eq. (41) give the best agreement with simulation, particularly for intermediate values of $n$. However, for small $n$ SAFTD underestimates the second virial coefficient more than TPT1-D and Eq. (39). All equations, however, tend to overestimate $B_{2}$ for large $n$. Considering that the only difference between SAFTD and TPT1-D is the quality of the correlation fit adopted for $g_{2}(\sigma)$, it is rather surprising that their predictions of $B_{2}$ are so different.

TABLE IV. Compressibility factor for the 32-mer hard-sphere fluid. Results from simulation and from various theories.

\begin{tabular}{lrrrrrrr}
\hline \hline \multicolumn{1}{c}{$\eta$} & $Z(\mathrm{MC})$ & GFD & TPT1 & TPT1-D & SAFTD & Eq. (39) & Eq. (41) \\
\hline 0.1021 & 7.22 & 8.85 & 9.17 & 8.12 & 7.58 & 7.52 & 7.23 \\
0.2017 & 23.26 & 25.65 & 26.65 & 24.58 & 24.32 & 23.67 & 23.01 \\
0.2504 & 37.5 & 39.5 & 41.1 & 38.5 & 38.5 & 37.6 & 36.8 \\
0.3013 & 58.9 & 60.3 & 62.7 & 59.6 & 59.7 & 58.9 & 57.9 \\
0.3505 & 88.9 & 89.1 & 92.7 & 89.2 & 89.1 & 88.9 & 87.8 \\
0.400 & 132.8 & 131.2 & 136.5 & 132.8 & 131.8 & 133.1 & 132.0 \\
0.449 & 199.3 & 192.4 & 200.3 & 196.8 & 193.7 & 197.7 & 196.9 \\
Average & $\%$ Error $=$ & 6.5 & 9.3 & 3.4 & 2.5 & 1.1 & 1.1 \\
\hline \hline
\end{tabular}




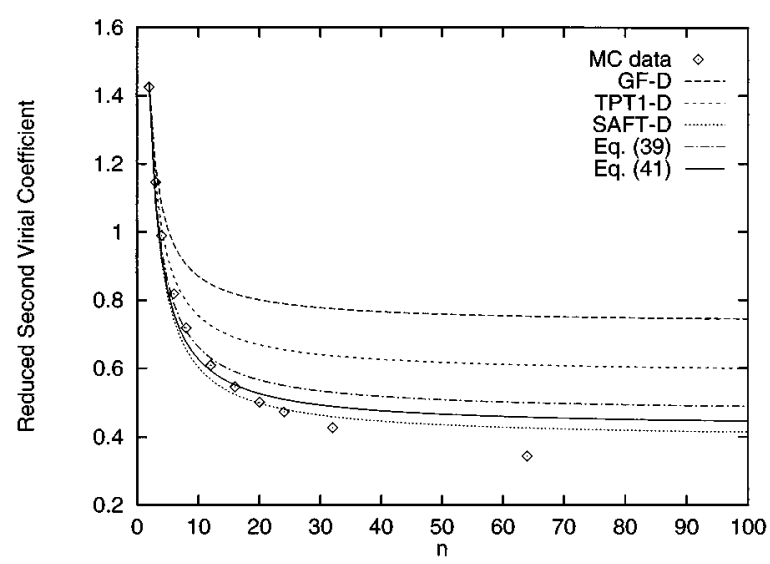

FIG. 4. Reduced second virial coefficient $\left(B_{2} / n^{2} \sigma^{3}\right)$ vs chain length from simulation (Ref. 26). The various lines correspond to predictions of several equations of state.

Further, for $n=4$ (when these two equations should be the very accurate), they predict $B_{2}$ inconsistently: TPT1-D overestimates the true value while SAFTD underestimates it significantly.

The second virial coefficient, being a measure of the slope of the $Z$ vs $\eta$ curve for $\eta \rightarrow 0$, seems to be very sensitive to the correlation constants adopted in the different models [i.e., for $Z(1), g_{1}(\sigma)$, etc.] For Eq. (39), the constants $c_{1}$ for $Z(2)$ and $Z(4)$ could be modified concertedly to provide a better agreement with $B_{2}$. Unfortunately, the scaling exponent of $B_{2}$ with chain length predicted by any equation of state of the form (36) [namely, Eq. (43)] is incorrect. ${ }^{18,26}$ Such a functional form is therefore unable to describe exact $B_{2}$ data over a wide range of $n$. Incidentally, correlation (40) does not predict the correct value of $B_{2}$ expected for an infinitely long chain $\left(B_{2}=0\right)$. Although alternative models for $P^{*}\left(r_{\infty}\right)$ can be postulated to correct this, the predicted values of $B_{2}$ for short chains would become inaccurate $\left[\alpha_{0}=0\right.$ in Eq. (43)]. In practice, however, accurate $B_{2}$ values are valuable when dealing with low-density fluids, composed predominantly of relatively short chains. In this context, Eq. (41) [with correlation (40)] provides a reasonable compromise (see Fig. 4) between accuracy and simplicity.

\section{EXTENSION TO MIXTURES}

A convenient reference system for description of blends of homopolymer chains (where the components can have different segment sizes) is the mixture of monomers that results from breaking the chains of the original mixture. This is because the volumetric properties of monomer mixtures of arbitrary size are well represented by the Mansoori et al. equation of state ${ }^{27}$ It is therefore convenient to first rewrite Eq. (35) including the chemical potential of the monomers:

$$
\mu^{E}(n)=n \mu(1)+\frac{n}{r}(r-1) \mu^{\text {bond }}(1)+\left(\frac{n}{r}-1\right) \mu^{\text {bond }}(r),
$$

$$
\begin{aligned}
\mu^{\text {bond }}(1) & =\frac{\mu^{E}(r)-r \mu^{E}(1)}{r-1}, \\
\mu^{\text {bond }}(r) & =\frac{\mu^{E}\left(r^{*}\right)-\frac{r^{*}}{r} \mu^{E}(r)}{\frac{r^{*}}{r}-1} .
\end{aligned}
$$

Equation (44) is now extended for component $i$ of a mixture as follows:

$$
\begin{aligned}
\mu^{E}\left(n_{i} \mid m\right)= & n_{i} \mu^{E}\left(1_{i} \mid 1\right)+\frac{n_{i}}{r_{i}}\left(r_{i}-1\right) \mu^{\text {bond }}\left(1_{i} \mid m\right) \\
& +\left(\frac{n_{i}}{r_{i}}-1\right) \mu^{\text {bond }}\left(r_{i} \mid m\right)
\end{aligned}
$$

where

$$
\begin{aligned}
\mu^{\text {bond }}\left(1_{i} \mid m\right) & =\frac{\mu^{E}\left(r_{i} \mid r\right)-r_{i} \mu^{E}\left(1_{i} \mid 1\right)}{r_{i}-1}, \\
\mu^{\text {bond }}\left(r_{i} \mid m\right) & =\frac{\mu^{E}\left(r_{i}^{*} \mid r^{*}\right)-\frac{r_{i}^{*}}{r_{i}} \mu^{E}\left(r_{i} \mid r\right)}{\frac{r_{i}^{*}}{r_{i}}-1},
\end{aligned}
$$

and where $\mu^{E}\left(n_{i} \mid m\right)$ denotes the excess chemical potential associated with the insertion of an $n_{i}$-mer into the real mixture $m$ and $\mu^{E}\left(r_{i} \mid r\right)$ is the chemical potential associated with the insertion of an $r_{i}$-mer into a reference mixture $r$. The reference mixtures denoted by $r$ and $r^{*}$ could be the same system but, in any case, they should resemble the mixture of interest $m$ (i.e., consistent in composition of site types, packing fraction, etc.) Substituting Eq. (47) into Eq. (12) we can write the resulting equation as follows:

$$
\begin{aligned}
Z(m)= & \bar{n} Z(1 \mid 1)+\sum_{i=1}^{N_{c}} \frac{x_{i} n_{i}}{r_{i}}\left(r_{i}-1\right) Z^{\text {bond }}\left(1_{i}\right) \\
& +\sum_{i=1}^{N_{c}} x_{i}\left(\frac{n_{i}}{r_{i}}-1\right) Z^{\text {bond }}\left(r_{i}\right),
\end{aligned}
$$

where

$$
\begin{aligned}
Z(1 \mid 1)= & 1+\frac{1}{\bar{n}} \sum_{i=1}^{N_{c}} n_{i} x_{i}\left[\beta \mu^{E}\left(1_{i} \mid 1\right)\right. \\
& \left.-\frac{1}{\eta} \int_{0}^{\eta} \beta \mu^{E}\left(1_{i} \mid 1\right) d \eta^{\prime}\right]
\end{aligned}
$$

and

$$
\begin{aligned}
Z^{\text {bond }}\left(p_{i}\right)= & -1+\beta \mu^{\text {bond }}\left(p_{i} \mid m\right) \\
& -\frac{1}{\eta} \int_{0}^{\eta} \beta \mu^{\text {bond }}\left(p_{i} \mid m\right) d \eta^{\prime} ; \quad p_{i}=1_{i}, \quad r_{i},
\end{aligned}
$$

$$
\bar{n}=\sum_{i=1}^{N_{c}} x_{i} n_{i}
$$

where 
$Z(1 \mid 1)$ is the compressibility factor of the hypothetical mixture of monomer segments that results from breaking the chain bonds of the real mixture (which can be evaluated by using the Mansoori et al. equation of state, ${ }^{27}$ and $\bar{n}$ is the molar average chain length.

In order to use Eq. (50) we need to know the insertion factors of $r_{i}$-mers and $r_{i}^{*}$-mers into the corresponding reference fluids. Given the numerous degrees of freedom of an arbitrary mixture (molecular lengths, geometry, concentration, etc.), these requirements limit severely the applicability of the approach proposed in this work. Some additional approximations are necessary; the crudest simplification that can be made is to assume that $\mu_{\text {bond }}\left(1_{i} \mid m\right)$ and $\mu_{\text {bond }}\left(r_{i} \mid m\right)$ are independent of the concentration and geometry of the molecules; in this case (which applies better to symmetric molecular systems such as freely jointed/tangent/equal-size hard sphere chains), from Eqs. (10), (48), (49), and (52) we can readily derive the approximations:

$$
\begin{aligned}
& Z^{\text {bond }}\left(1_{i}\right)=Z_{\sigma=\sigma}^{\text {bond }}(1)=\frac{Z\left(r_{i}\right)-r_{i} Z(1)}{r_{i}-1}, \\
& Z^{\text {bond }}\left(r_{i}\right)=Z_{\sigma=\bar{\sigma}}^{\text {bond }}\left(r_{i}\right)=\frac{r_{i} Z\left(r_{i}^{*}\right)-r_{i}^{*} Z\left(r_{i}\right)}{r_{i}^{*}-r_{i}} .
\end{aligned}
$$

Equations (54) and (55) are expected to provide good results if the diameter of a given monomer unit is close to an average diameter for the system, $\bar{\sigma}$, e.g.,

$$
\bar{\sigma}=\frac{\sum_{i=1}^{N_{c}} x_{i} n_{i} \sigma_{i}^{3}}{\sum_{i=1}^{N_{c}} x_{i} n_{i} \sigma_{i}^{2}} .
$$

The diameter $\bar{\sigma}$, a natural size-scaling parameter in the theory of hard-sphere mixtures, ${ }^{27}$ is chosen here because it approaches $\sigma_{i}$ whenever the latter becomes the dominant length in the system.

For true homonuclear chains $Z(1 \mid 1)=Z(1)$ and if the same reference fluids are used for all components, Eq. (50) with approximations (54) and (55) can then be simplified to

$$
Z=Z(r)+\frac{\bar{n}-r}{r^{*}-r}\left[Z\left(r^{*}\right)-Z(r)\right] .
$$

Comparing Eqs. (57) and (36) it follows that the compressibility factor of a polydisperse mixture is equivalent to that of a monodisperse system of chain length $\bar{n}$

$$
Z=Z(\bar{n})=\sum_{i=1}^{N_{c}} x_{i} Z\left(n_{i}\right) .
$$

This result has been shown before to hold (under certain conditions) for the GF theory ${ }^{2,15}$ and in general for the TPT1 theory. ${ }^{5}$

For systems with components of different-size monomers, the use of Eq. (50) is more involved. Other reference systems besides the hard-sphere mixture need to be studied or its properties must be estimated by introducing suitable assumptions. ${ }^{6,15,28}$ Preliminary calculations for this type of systems using Eqs. (50) and (54) and (55) show that fair agreement is obtained with simulation data. However, larger

\begin{tabular}{|c|c|c|c|c|c|c|c|}
\hline$x_{1}$ & $\sigma_{2} / \sigma_{1}$ & $\begin{array}{c}\text { MC data } \\
\text { (Ref.) }\end{array}$ & Eq. (58) & GF & TPT1 & TPT1-D & Eqs. $(50),(60)$ \\
\hline 0.12 & 1.0 & 2 & 1.90 & 6.00 & 3.29 & 2.27 & 1.96 \\
\hline 0.33 & 1.0 & 27 & 0.20 & 9.12 & 3.61 & 1.17 & 0.25 \\
\hline 0.33 & 1.25 & 27 & 0.96 & 8.79 & 3.08 & 0.82 & 0.39 \\
\hline 0.10 & 0.50 & 15 & 17.58 & 2.93 & 1.96 & 2.12 & 1.78 \\
\hline 0.60 & 2.0 & 15 & 10.65 & 11.37 & 3.27 & 2.38 & 1.96 \\
\hline 0.12 & 4.0 & 15 & 27.31 & 6.65 & 1.50 & 0.45 & 1.58 \\
\hline
\end{tabular}
deviations are observed when the difference in sizes of the
TABLE V. Average relative error $(\%)$ in the compressibility factor of mixtures of 8 -mer/monomer hard-sphere systems from various theories. Subindexes 1 and 2 refer to the 8 -mer and monomer, respectively.

monomeric units that make up the different chains becomes large (say >2). A simple correction to these results is described next.

In contrast to the uniform segment size case, another limiting case arises when a component has a monomer diameter negligible compared with that of the other monomers. The corresponding equation for $Z^{\text {bond }}$ can be estimated as follows. If $\sigma_{1} / \sigma_{2} \rightarrow 0$, the compressibility factor of a system of $N_{1}, n_{1}$-mers, and $N_{2}$ monomers as computed from Eq. (50) [letting $Z^{\text {bond }}\left(1_{i}\right)=Z^{\text {bond }}\left(r_{i}\right)$ ] should be equal to the compressibility factor of a system of $N_{1}$ "point" monomers and $N_{2}$ monomers as evaluated from the Mansoori et al. equation of state. ${ }^{27}$ The resulting equation can be simplified to yield

$$
Z_{\sigma_{1} / \sigma_{2} \rightarrow 0}^{\text {bond }}=\frac{1}{1-\eta} .
$$

Incidentally, the same result can be obtained by taking the appropriate limit of the corresponding expression for $Z^{\text {bond }}$ derived from the TPT1 theory for mixtures. Equation (55) provides a good estimate of $Z^{\text {bond }}$ for $\sigma_{i} / \bar{\sigma} \rightarrow 1$, while Eq. (59) is appropriate when $\sigma_{i} / \bar{\sigma} \rightarrow 0$; we therefore propose a simple interpolation scheme according to

$$
\begin{gathered}
Z^{\text {bond }}\left(p_{i}\right)=\frac{\sigma_{i}}{\bar{\sigma}} Z_{\sigma=\bar{\sigma}}^{\text {bond }}\left(p_{i}\right)+\left(1-\frac{\sigma_{i}}{\bar{\sigma}}\right) \frac{1}{1-\eta} ; \\
p_{i}=1_{i}, r_{i} .
\end{gathered}
$$

Table $\mathrm{V}$ summarizes a comparison of the predictions of Eqs. $(50) /(60)$ (for $\left.r=2, r^{*}=4\right)$ to simulation results for systems of 8-mer/monomer reported by various authors for different concentrations, densities, and sizes of monomeric units. ${ }^{2,15,29}$ For comparison, we include the average relative errors obtained by using the simple conformal solution Eq. (58) (using correlation (17) with the constants of Table I for the monomer and the 8-mer), the GF theory as extended by Wichert and Hall ${ }^{15}$ to mixtures, the TPT1 theory, and a recent extension of TPT1-D to mixtures. ${ }^{29}$ Overall, Eqs. (50)/ (60) and the TPT1-D theory of Chang and Sandler yield the best results. The conformal equation of state (58) and Eqs. $(50) /(60)$ give more accurate results than the TPT1-D for chain/monomer mixtures of segments of equal diameter, much like in the case of homopolymer systems. As expected, the conformal mixing rule becomes increasingly inaccurate as the ratio of segment diameters of both components diverges from unity. In spite of the complicated algebra in- 


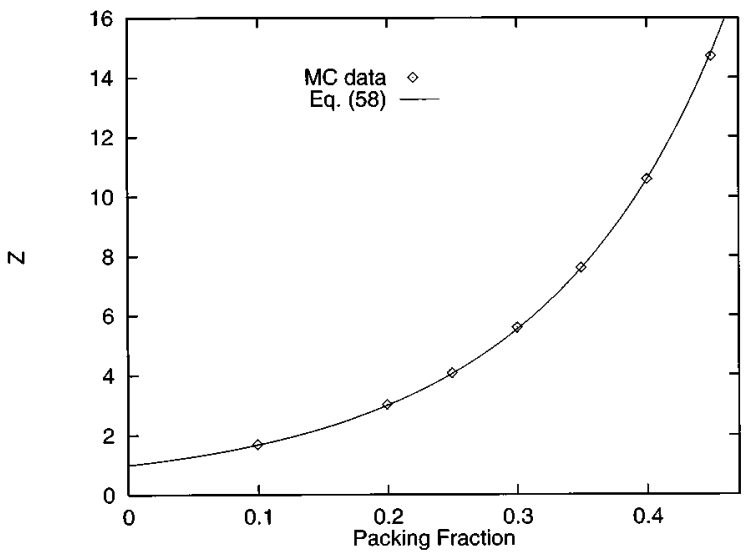

FIG. 5. Compressibility factor vs packing fraction for a concentrated solution of 16-mers dissolved in the monomer (50\% volume). The diamonds show the results of MC simulations (Ref. 11) and the full line corresponds to the predictions of Eq. (58).

volved in its use, the GF equation of state for mixtures is significantly less accurate than the TPT1, TPT1-D, and Eqs. $(50) /(60)$.

The results of simulations of mixtures for short chains or mixtures of short chains and monomer of uniform segment size seem to be well represented by most of the preceding equations of state (i.e., the conformal mixing rule). This is further confirmed in Fig. 5 which compares simulation data for a $50 \%$ volume 16 -mer/monomer system ${ }^{11}$ to the predictions of Eq. (58). Similar behavior is observed for predictions from the TPT1, TPT1-D, or Eqs. (50)/(60).

\section{EXTENSION TO MORE COMPLEX SYSTEMS}

The approach illustrated by Eqs. (50) and (60) of the previous section can be easily extended to the case of mixtures of heteronuclear, tangent or fused hard sphere chain systems (i.e., including copolymers). For this we only need to build into the model equations of state for suitable reference systems. For example, a number of equations of state for short fused chains with asymmetric sites have been proposed $^{28,30,31}$ which could be incorporated to the model. This issue is being examined in more detail to arrive at definite recommendations. Further, the study of molecular models described by soft intermolecular potentials could also be undertaken using our approach once the properties of the relevant reference systems have been generated. The TPT1 formalism or the GF theory in any of their variations are less flexible for application to more complex systems.

Branched polymers represent another important class of polymer systems. The intramolecular component of a branched-chain free energy will differ from that of a linear chain since the sampling space for inserting sites in the neighborhood of the branching points is restricted by steric hindrances. However, for purely repulsive chains, this intramolecular component does not directly affect the equation of state, i.e., any density-independent term of $\mu^{E}$ cancels out when evaluating $Z$ from Eq. (10). Branching will significantly affect the packing properties of the system when sites of different sizes are part of the same chain or if rigid con-

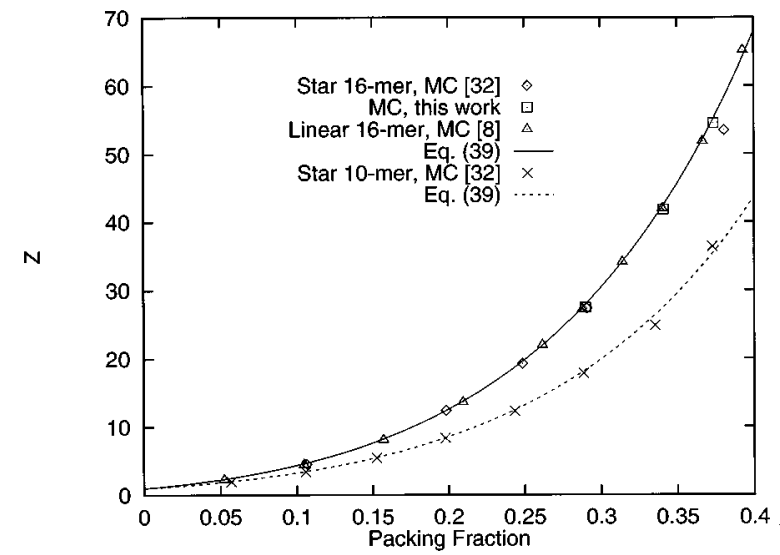

FIG. 6. Compressibility factor vs packing fraction for hard-core, 3-arm star polymers. The diamonds (16-mer) and $\times$ 's (10-mer) show results of simulations from Ref. 32, and the squares show the results of the simulations performed in this work. For the linear hard-core chains, the triangles show results of simulations for the 16-mers from Ref. 32, and the lines show the predictions of Eq. (39).

straints are associated to branching points. The shielding of branching points and the prevalence of more compact molecular arrangements also affect the pressure of the system. All these effects are basically a result of intermolecular interactions.

Let us consider branched/tangent/equal-size hard-sphere chains with 3-arm branching points located far enough from each other so that sites on different branches are uncorrelated. We can assume that the $\mu^{E}$ (append a monomer) term of Eq. (32) is uniform throughout the chain, except for the first sites branching from the main chain section. The $\mu^{E}$ (append a branch-monomer) term will differ from the usual $\mu^{E} \quad$ (append a monomer) by approximately $\left[\mu^{E}\left(r^{\prime}\right.\right.$-branch $\left.)-\mu^{E}\left(r^{\prime}\right)\right] / n_{\mathrm{sb}}$, where $r^{\prime}$ branch is a (short) branched reference system with total sites equal to that of an $r^{\prime}$-mer linear reference system and $n_{\mathrm{sb}}$ is the number of sites in the lateral branches of $r$ branch. A simple illustration can be implemented for the dimer-tetramer equation of state (39); if we correlate the compressibility factor for the isotetramer hard chain $\left(4^{*}\right)$, i.e., $r^{\prime}=4, n_{\mathrm{sb}}=1$; we can then arrive at the following equation for an $n$-mer chain with $n_{b}$ branches:

$$
\begin{aligned}
Z\left(n, n_{b}\right)= & Z(2)+(n-r) \frac{Z(4)-Z(2)}{2} \\
& +n_{b}\left[Z\left(4^{*}\right)-Z(4)\right] .
\end{aligned}
$$

Our simulations reveal, however, that $Z\left(4^{*}\right)$ does not differ appreciably from $Z(4)$, thus $Z\left(n, n_{b}\right) \sim Z(n)$, i.e., the pressure of linear and branched polymers of equal number of sites at the same density is comparable. Simulation results for 3-arm star chains of 10 and 16 sites and 4-armed star chains of 13 and 21 sites (Yethiraj and Hall) ${ }^{32}$ support the fact that the pressure of repulsive chain systems is not a strong function of the details of the molecular architecture. In Fig. 6 we show simulation data for the 3-arm chains and the predictions of Eq. (39) for the corresponding linear 
chains. It is observed that the agreement with previous simulation data ${ }^{32}$ is very good except at the higher densities $(\eta$ $>0.30$ ). Since such behavior is difficult to explain, we performed simulations for the 16-mer star system (using the ECCB method) ${ }^{11}$ for $\eta>0.28$. Our results are in better agreement with Eq. (39). Since our simulations rely on MC methods specifically designed to efficiently handle dense branched systems, we are inclined to believe that our simulation results are more accurate. We thereby conclude that no divergent trend occurs at high densities.

As discussed by Yethiraj and Hall ${ }^{32}$ the effect of branching on the pressure of the system will be more pronounced for molecules with attractive interactions, in which case the approach illustrated by Eq. (61) can prove to be more useful. Such an approach can also be exploited when dealing with branched systems, where the branching points are modeled with rigid constraints or with dissimilar site sizes.

\section{CONCLUSIONS}

In this work we have explored the connection between the GF- $r$ and TPT1- $r$ theories in order to formulate a more general model for the equation of state of freely jointed hardsphere chain molecules. The model is first expressed in terms of the chemical potential and its basic assumptions are justified in light of our recent simulations of the chemical potential.

From our general model, two alternative equations of state are proposed based on particular choices of the reference fluids: one is based on the dimer/tetramer fluids and the other on the dimer fluid and an infinitely long chain fluid. Both equations are shown to accurately reproduce simulation data for homopolymer hard-sphere systems for chain lengths ranging from $n=3$ up to 51 . The performance of both equations is comparable, and they are more accurate than other available equations of state. It is also shown that the same general model for the equation of state is not capable of predicting the correct scaling exponent of the second virial coefficient with chain length. Nevertheless, the equations proposed in this work give good estimates of the second virial coefficient for short chains (i.e., $n<25$ ).

Our approach has been also extended to mixtures of homopolymer chains with components of arbitrary segment size. For the case of uniform segment size, the equation of state reduces to the conformal mixing rule. For the case of nonuniform segment size an equation is derived based on simple heuristic arguments. When compared to simulation data for a number of 8 -mer/monomer systems, the equation proposed in this work is shown to provide results with accuracy comparable to that of equations of state derived from the TPT1 theory and superior to those derived from the GF theory for mixtures.

Other extensions of our approach to more complex systems are also discussed. In particular, the application to branched polymers is introduced; our derivations suggest that the pressure of typical, simple branched systems is comparable to that for linear chains with the same total number of sites per chain. This prediction is confirmed by simulation data for two star systems.

A number of simulations are currently under way to further test all the theories discussed in this work, particularly for complex systems consisting of highly asymmetric components and more elaborated models of branched polymers and copolymers.

\section{ACKNOWLEDGMENTS}

The authors gratefully acknowledge the financial support of the National Science Foundation and from the industrial sponsors of the University of Wisconsin Polymerization Reaction Engineering Laboratory. The authors are also grateful to the Petroleum Research Fund of the American Chemical Society.

${ }^{1}$ R. Dickman and C. K. Hall, J. Chem. Phys. 85, 4108 (1986).

${ }^{2}$ K. G. Honnell and C. K. Hall, J. Chem. Phys. 90, 1841 (1989).

${ }^{3}$ M. S. Wertheim, J. Chem. Phys. 85, 2929 (1986).

${ }^{4}$ M. S. Wertheim, J. Chem. Phys. 87, 7323 (1987).

${ }^{5}$ W. G. Chapman, G. Jackson, and K. E. Gubbins, Mol. Phys. 65, 1057 (1988)

${ }^{6}$ Y. C. Chiew, Mol. Phys. 70, 129 (1990).

${ }^{7}$ D. Ghonasgi and W. Chapman, J. Chem. Phys. 100, 6633 (1994).

${ }^{8}$ J. Chang and S. I. Sandler, Chem. Eng. Sci. 49, 2777 (1994).

${ }^{9}$ P. J. Flory, J. Chem. Phys. 10, 51 (1942).

${ }^{10}$ M. L. Huggins, Ann. N. Y. Acad. Sci. 43, 1 (1942).

${ }^{11}$ F. A. Escobedo and J. J. de Pablo, J. Chem. Phys. 102, 2636 (1995). Note that in Table II of this reference the packing fraction of the 16-mer/ monomer system at $P^{*}=6.7$ was incorrectly reported to be 0.437 . The correct value is 0.447 .

${ }^{12}$ A. Yethiraj and C. K. Hall, Mol. Phys. 80, 469 (1993).

${ }^{13}$ S. Phan, L. Kierlik, M. L. Rosinberg, H. Yu, and G. Stell, J. Chem. Phys. 99, 5326 (1993)

${ }^{14}$ K. G. Honnell and C. K. Hall, J. Chem. Phys. 95, 4481 (1991).

${ }^{15}$ J. M. Wichert and C. K. Hall, Chem. Eng. Sci. 49, 2793 (1994).

${ }^{16}$ N. F. Carnahan and K. E. Starling, J. Chem. Phys. 51, 635 (1969).

${ }^{17}$ D. J. Tildesley and W. B. Streett, Mol. Phys. 41, 341 (1980).

${ }^{18}$ C. P. Bokis, Y. Cui, and M. D. Donohue, J. Chem. Phys. 98, 5023 (1993).

${ }^{19}$ Y. C. Chiew, Mol. Phys. 73, 359 (1991).

${ }^{20}$ A. Yethiraj and C. K. Hall, J. Chem. Phys. 93, 5315 (1990).

${ }^{21}$ F. A. Escobedo and J. J. de Pablo, J. Chem. Phys. (in press).

${ }^{22}$ R. Dickman and C. K. Hall, J. Chem. Phys. 89, 3168 (1988).

${ }^{23}$ E. A. Muller and K. Gubbins, Mol. Phys. 80, 957 (1993).

${ }^{24}$ F. A. Escobedo and J. J. de Pablo, Macromol. Chem. Phys. Theory Sim. (in press).

${ }^{25}$ M. A. Denlinger and C. K. Hall, Mol. Phys. 71, 541 (1990).

${ }^{26}$ A. Yethiraj, K. G. Honnell, and C. K. Hall, Macromolecules 25, 3979 (1992)

${ }^{27}$ G. A. Mansoori, N. F. Carnahan, K. E. Starling, and T. W. Leland, Jr., J. Chem. Phys. 54, 1523 (1971).

${ }^{28}$ M. D. Amos and G. Jackson, J. Chem. Phys. 96, 4604 (1992).

${ }^{29}$ J. Chang and S. I. Sandler (preprint).

${ }^{30}$ A. L. Archer and G. Jackson, Mol. Phys. 73, 881 (1991).

${ }^{31}$ S. Phan, E. Kierlik, and M. L. Rosingberg, J. Chem. Phys. 101, 7997 (1994)

${ }^{32}$ A. Yethiraj and C. K. Hall, J. Chem. Phys. 94, 3943 (1991). 